\title{
Las trayectorias educativas de hombres y mujeres jóvenes. Una aproximación desde el análisis de secuencias
}

\author{
Albert Julià \\ Universidad de Barcelona. Departamento de Sociología \\ albert.julia.cano@ub.edu
}

\section{Resumen}

Sobre la base de la Encuesta a la Juventud de Cataluña 2012, en este artículo se define una tipología de trayectorias educativas de los jóvenes para discernir cuáles son los factores que determinan los itinerarios académicos y en qué medida existen desigualdades significativas de género. Mediante el estudio de secuencias y el análisis clúster, se determinan tres categorías de trayectorias educativas ("voluntariosos», "exitosos» $\mathrm{y}$ "desertores») que plasman el papel configurador de los factores sociodemográficos analizados. Los resultados obtenidos indican que ser mujer reduce la probabilidad de pertenecer a un itinerario con un alto grado de abandono educativo temprano, y que este se produce más a menudo entre los autóctonos que entre los jóvenes de origen inmigrante. Las mujeres cuyos padres han conseguido grados académicos elevados permanecen en mayor medida en el sistema educativo que los chicos. Los niveles altos de apoyo parental constituyen una mayor garantía en cuanto a la continuidad en los estudios de los jóvenes que el apoyo que proporcionan profesores o tutores.

Palabras clave: trayectorias educativas, juventud, género, análisis de secuencias, análisis clúster

Abstract. Educational pathways of young men and women. A sequence analysis approach

This article defines a typology of youth educational trajectories to ascertain which factors determine the educational pathways and the extent of significant gender inequalities. With the Survey of Youth of Catalonia 2012 data, and using sequence and cluster analyses, we find three distinct categories ("willful», "successful» and "deserter») which determine the shape and the role of socio-demographic factors. The reductive effect of being a woman on the probability to belong on a trajectory with a high level of early school dropout occurs to a greater extent in the native population, while the effect of gender in immigrants is lower. Women are positively influenced more than men by higher educational levels of their parents. We find a high level of parental support is the greatest guarantor of continuity in studies of young people than the support given by teachers or tutors.

Keywords: Educational trajectories, youth, gender, sequence analysis, cluster analysis 


\section{Sumario}

1. Introducción 4. Resultados

2. Factores asociados a la brecha de género y las trayectorias educativas

3. Base de datos y metodología

Apéndice

Referencias bibliográficas

\section{Introducción}

Las trayectorias académicas de los jóvenes en España son diversas y contrapuestas. Mientras en los últimos años aumenta la proporción de alumnos inscritos en la universidad, aún persiste en el sistema educativo español un porcentaje considerable de jóvenes que abandonan prematuramente los estudios. Esta pauta tiene importantes consecuencias en sus trayectorias vitales. Los estudiantes que retrasan su entrada en la educación postobligatoria o en la universidad, como aquellos que interrumpen temporalmente alguno de estos niveles educativos, terminan por permanecer menos años en el sistema educativo y obtienen títulos de niveles más bajos que aquellos que mantienen una continuidad en sus itinerarios académicos (Featherman y Carter, 1976; Bozick y DeLuca, 2005; Desjardins et al., 2006). Los estudiantes con mayores desventajas socioeconómicas y de capital cultural y social tienden a mostrar trayectorias más discontinuas que repercuten negativamente en las probabilidades de obtener niveles superiores al de secundaria obligatoria o de llegar a la universidad (Milesi, 2010).

Por trayectorias educativas entendemos al conjunto de transiciones entre los diferentes niveles académicos, el tiempo dedicado en cada etapa y el orden en que se suceden los distintos estados educativos de los jóvenes (si están estudiando o no y en qué nivel se encuentran). Las diferencias en los itinerarios pedagógicos están asociadas a múltiples factores. La brecha de género ha sido uno de los elementos que han centrado buena parte del estudio de las diferencias entre los rendimientos y las trayectorias académicas (Hausman et al., 2009; Jacob, 2002). Las diferencias de género son apreciables en distintos indicadores desde las primeras etapas educativas. En España, el 86,3\% de las chicas de 12 años ya han completado la educación primaria, mientras que en el caso de los chicos se trata del $81,5 \%$ (la brecha se detecta en todas las comunidades autónomas). Los estudiantes varones que alcanzan cuarto de ESO con 15 años representan el 56,9\%, mientras que en las mujeres es el 66,8\% (web del Ministerio de Educación, Cultura y Deporte, curso 2011-2012).

En España se ha reducido en los últimos años el porcentaje de jóvenes de 18 a 24 años que abandonan prematuramente el sistema educativo, pero se mantiene muy por encima de la media europea. El abandono académico temprano se define como el porcentaje de la población de 18 a 24 años que tiene como máximo el título de enseñanza secundaria obligatoria (en la actualidad, la ESO en España) y no está cursando ningún tipo de formación (Eurostat). En 2013, 
era en España el 23,6\%, y en la UE27, el 12\% (datos de Eurostat). Algunos autores señalan que dicha disminución se ha producido esencialmente por la reducción de oportunidades laborales que el mercado ofrece a los jóvenes en trabajos de baja cualificación, especialmente para los hombres, ya que el abandono masculino está más correlacionado con la incorporación al mercado de trabajo que el femenino (Fernández-Enguita et al., 2010). La mayoría de indicadores pedagógicos en los últimos años evidencian que las mujeres representan cada vez más la cara del éxito académico. Frente a ellas, el avance de los varones es mucho más modesto y, como consecuencia de ello, la concentración del fracaso educativo en este colectivo es más evidente. Examinando la situación de los jóvenes de 18 a 24 años sin titulación postobligatoria, las diferencias son muy significativas. Según datos del Instituto Nacional de Evaluación Educativa de 2014 (Ministerio de Educación, Cultura y Deporte), el 27\% de los varones y el 19,9\% de las mujeres han abandonado su formación de forma temprana. Asimismo, se encuentran ciertas similitudes a nivel universitario. Si bien las diferencias por género de las matriculaciones universitarias dependen en gran medida de la rama de que se trate (en ingeniería y arquitectura se mantiene un porcentaje más elevado de hombres), en general, encontramos una mayor proporción de mujeres que llegan más lejos en sus estudios. El 54,3\% de los estudiantes matriculados son mujeres y, en el caso de los egresados, la cifra aumenta en más de 3 puntos porcentuales (un 57,4\% de mujeres) (web del Ministerio de Educación, Cultura y Deporte, curso 2012-2013). Algunas investigaciones atribuyen esta brecha de género, que se observa en todas las sociedades occidentales, al mayor atractivo que supone para ellas llegar a niveles educativos superiores (Mortenson, 1999). Para algunas mujeres jóvenes, el hecho de continuar con los estudios supone una forma de postergar o de evitar la asunción de roles tradicionales de género respecto a la división del trabajo doméstico. En este sentido, la división sexual del trabajo doméstico incentiva de forma diferenciada a los hombres y a las mujeres, ya que ellos no se sienten presionados a dedicarse a la labor de cuidadores o a otras tareas del hogar como algunas mujeres (Martínez García, 2007).

Algunas investigaciones han centrado su explicación sobre la brecha educativa de género en las diferencias existentes entre los dos sexos acerca de ciertas habilidades no cognitivas que se asocian al éxito académico, como, por ejemplo: la atención, la persistencia, el afán de aprender o la capacidad de trabajar de forma independiente (Jacob, 2002). Algunos autores, como Martínez García (2011), señalan que, aunque hay una brecha de género consistente tanto en competencias como en resultados académicos, es en los resultados donde se aprecian diferencias más significativas.

La mayoría de datos analizados entorno a los indicadores pedagógicos tienen un fuerte carácter transversal, y son escasos los análisis orientados hacia un estudio longitudinal. En este sentido, la presente investigación se propone ir más allá del examen de meros datos transversales y plantea como principal objetivo establecer una tipología de itinerarios educativos de los jóvenes que permita analizar los factores que se asocian a las diferentes trayectorias. Mediante esta tipología se analiza cómo estos factores minimizan o incrementan el 
efecto que deriva del sexo y en qué tipo de trayectorias resultan significativas. De esta forma se identifican los elementos que potencian o merman la asociación entre el sexo de los jóvenes y el de recorridos educativos. Para examinar los diferentes itinerarios académicos se utiliza el análisis de secuencias como metodología de estudio temporal. Se trata de un recurso metodológico hasta ahora poco utilizado en las ciencias sociales en España.

\section{Factores asociados a la brecha de género y las trayectorias educativas}

Existe cierto nivel de consenso en la literatura especializada que señala que una de las claves del peor rendimiento educativo en los varones reside en déficits de habilidades no cognitivas. Los individuos que no poseen este tipo de habilidades tienden a desarrollar carreras educativas más pobres. Según el estudio de Heckman et al. (2006), en el que cuantifican el valor relativo de habilidades cognitivas y no cognitivas, cuanto mayores son las habilidades no cognitivas en edades tempranas, más probable es que las personas obtengan un título universitario o que tengan un salario más elevado en edades más avanzadas. A su vez, es menos probable que desarrollen conductas disruptivas que conducen, en el caso de los hombres, a problemas con la justicia y el sistema penal y, en el de las mujeres, a ser madres solteras adolescentes.

La literatura señala que los chicos tienden a presentar mayores carencias respecto a habilidades interpersonales y de comunicación, son menos persistentes y menos disciplinados que las chicas (Jacob, 2002; McFadden et al., 1992; Skiba et al., 1997) y tienen una mayor probabilidad de desarrollar comportamientos antisociales (Moffitt et al., 2001) o de ser castigados en la escuela. Los resultados de las investigaciones de Cooley (1995) y Gregory (1996) muestran que los chicos tienen hasta cuatro veces más probabilidades de ser castigados en la escuela que las chicas. Los déficits en estas habilidades han sido relacionados con recorridos académicos más cortos. Como apunta Freeman (2004), las habilidades no cognitivas están intrínsecamente relacionadas con la mayor probabilidad que tienen los chicos de repetir curso. En contraste, las mujeres presentan mejores condiciones en relación con las habilidades no cognitivas como la atención, las habilidades organizativas (Jacob, 2002) o la autodisciplina (Duckworth et al., 2006), y un mayor interés en la escuela y en los estudios (Jacob, 2002; Rosenbaum, 2001). Ellas presentan una mayor atracción por llegar a niveles educativos superiores, debido a que suelen estar más vinculadas a la educación (Mortenson, 1999), responden en mayor medida que les gusta estudiar y muestran mayor conocimiento acerca de cómo su futuro éxito en la universidad y el trabajo está directamente relacionado con su esfuerzo académico en secundaria (DiPrete y Buchmann, 2013).

La amplia literatura publicada sobre la influencia de las habilidades no cognitivas asociadas a la brecha de género en rendimientos académicos indica que las trayectorias (no solo) educativas vienen determinadas en gran medida por el sexo de las personas. En este sentido, la primera hipótesis vendría a confirmar la relación entre género y logro académico: 
- Hipótesis 1 (H1): El género es un factor predictivo de las trayectorias educativas de los jóvenes.

Sin embargo, hay abundantes investigaciones que han asociado tanto el rendimiento académico como las diferencias derivadas del sexo de los estudiantes con otros factores de consensuada relevancia. Un elemento destacado para algunas investigaciones es la asociación del origen de los alumnos en el rendimiento educativo (Calero y Choi, 2009). Análisis como el de Calero y Waisgrais (2008) ponen de relieve el menor rendimiento de los estudiantes de origen inmigrante en comparación con los autóctonos. Las diferencias de origen también tienen sus derivaciones en la brecha de género y en las trayectorias pedagógicas. Según algunas investigaciones, los hombres inmigrantes tienen expectativas educativas y ocupacionales más bajas que las mujeres cuando realizan estudios secundarios, y también son menos propensos a desarrollar altas expectativas educativas cuando se acercan a la adultez (Portes y Rumbaut, 2001; Feliciano y Rumbaut, 2005; Escandell et al., 2015). A raíz de estas investigaciones, cabe esperar que:

- Hipótesis $2(\mathrm{H} 2)$ : El origen tenga un impacto en los itinerarios educativos de los jóvenes, y que la asociación de los jóvenes inmigrantes con las trayectorias educativas venga influenciada a su vez por el sexo.

La literatura sobre el rendimiento de los estudiantes ha demostrado de forma consistente que el nivel educativo de los padres es importante en la predicción del logro y en las trayectorias educativas de los hijos e hijas (DavisKean, 2005; Smith et al., 1997; Zhu et al., 2014). Las expectativas de los estudiantes sobre su permanencia o su abandono del sistema educativo, o si realizan o no estudios superiores, tiene implicaciones importantes según los tipos de estimulación proporcionados en el hogar (Davis-Kean, 2005). Las expectativas y el apoyo parental resultan claves para entender las aspiraciones académicas de los hijos e hijas y sus itinerarios educativos (Mau, 1997; McBride y Lin, 1996; Muller, 1998). Para Buchmann et al. (2008), los estilos parentales y las expectativas son responsables de las personalidades y de los comportamientos de los hijos e hijas, y acentúan la brecha educativa de género. Sin embargo, a pesar de que algunos estudios encuentran evidencias de la influencia del apoyo parental en el logro educativo, estos apenas observan diferencias significativas entre chicos y chicas (Muller, 1998; Jeynes, 2005). A tenor de estas investigaciones, se plantean las siguientes hipótesis:

- Hipótesis 3 (H3): El nivel educativo de los padres está asociado a las trayectorias de los chicos y chicas de forma diferenciada.

- Hipótesis 4 (H4): El apoyo parental influye en las trayectorias de los jóvenes también de forma desagregada por sexos.

Otro tipo de investigaciones han puesto el foco de atención en la importancia que tiene la escuela y, en especial, el apoyo que reciben los estudiantes 
por parte de sus referentes académicos como determinantes de los itinerarios educativos (Rubie-Davies, 2007; 2010; Sorhagen, 2013; Weinstein y McKown, 1998). Profesores y tutores pueden llegar a ejercer una influencia clave en algunos alumnos a la hora de decidir las vías educativas futuras. Para Farkas et al. (1990) las expectativas de los maestros también pueden venir determinadas por diferencias de sexo en lo que definen como el sesgo del maestro, que surge en parte por las percepciones de los docentes y el efecto de las profecías autocumplidas sobre el rendimiento de los estudiantes. Según esta teoría, los profesores tienden a favorecer a aquellos perfiles que consideran que suelen tener mejores rendimientos. En este sentido, para Entwisle et al. (2007), en los últimos años, el sesgo del docente jugaría a favor de las mujeres (en dirección opuesta a los resultados de Farkas et al. (1990)), debido a la amplia sobrerrepresentación femenina. En la misma línea, otros estudios longitudinales apuntan que los chicos logran mejores puntuaciones cuando es un profesor el que imparte clases y no una profesora (Sokal et al., 2007). Sin embargo, la evidencia empírica de estas investigaciones no suele ser concluyente (Buchmann et al., 2008). Siguiendo las postulaciones de Entwisle et al. (2007), se plantea la siguiente hipótesis:

- Hipótesis 5 (H5): Las chicas reciben un efecto mayor del apoyo de los docentes o referentes académicos para obtener trayectorias educativas más duraderas y que llevan a un mayor éxito educativo que los chicos.

\section{Base de datos y metodología}

Para realizar los análisis se ha utilizado la Encuesta a la Juventud de Cataluña 2012 (EJC12). Esta base de datos consta de una muestra representativa de 3.002 jóvenes catalanes con edades comprendidas entre los 15 y los 34 años. La encuesta contiene datos retrospectivos sobre los recorridos formativos. El análisis se centra en los itinerarios que han seguido los jóvenes desde los 15 hasta los 30 años. Para poder obtener las trayectorias completas de estos periodos de edad, los análisis se llevan a cabo con los individuos con edades comprendidas entre los 30 y los 34 años ( $n=944$, el 31,4\% de la muestra inicial), es decir, los nacidos entre 1978 y 1982. De esta manera se pueden comparar los itinerarios de individuos que han transitado durante el mismo periodo temporal (desde los 15 hasta los 30 años). La información de su situación en relación con el sistema educativo se obtiene mediante las fechas (mes y año) en que se inicia cada nivel o especialidad de estudios hasta su finalización, interrupción, cambio de especialidad o abandono. Una vez realizada la equivalencia de fechas en mediciones temporales se disponen los puntos temporales. Los puntos temporales se han dispuesto en cuatrimestres. Esto supone 46 puntos temporales (desde la situación de los individuos del primer cuatrimestre a los 15 años hasta la situación del primer cuatrimestre a los 30 años).

$\mathrm{El}$ análisis propuesto en este estudio se realiza en dos etapas. En la primera fase, se determina la secuencia de trayectorias de los jóvenes mediante el análisis de correspondencias óptimas y se estudia si el género es un factor discrimina- 
torio de los itinerarios educativos. Alguna investigación reciente ha realizado alguna propuesta de trayectorias educativas sobre el análisis de secuencias utilizando la EJC12 (Troiano y Daza, 2016). Una vez identificadas las secuencias para cada individuo, se presenta el análisis de conglomerados con el objetivo de crear una tipología de jóvenes a partir de las diferencias y similitudes de las trayectorias educativas. En esta segunda fase se realizan diferentes modelos de regresión logística multinomial que permiten comparar cuáles son las características de los jóvenes asociadas a las diferentes tipologías de trayectorias educativas y testar las diferentes hipótesis previamente planteadas mediante las interacciones de las diferentes variables con el género. Los estudios se han llevado a cabo a partir del programa estadístico $R$ y mediante el paquete TraMineR (ver Gabadinho et al., 2009, 2011).

\subsection{El análisis de secuencias}

Uno de los atractivos de esta investigación es poder desarrollar una metodología poco utilizada en las ciencias sociales en España, pero presente en algunos estudios anglosajones. El análisis de secuencias goza de mayor reconocimiento y ha sido empleado con considerable frecuencia en el ámbito de la biología — principalmente en estudios de genética— (Sankoff y Kruskal, 1983; Abbott y Tsay, 2000). En el análisis de secuencias cada trayectoria del ciclo de vida se representa como una cadena de caracteres (también numérica), similar a la utilizada para codificar las moléculas de ADN en las ciencias biológicas (Aassve et al., 2007). Para estudiar las secuencias de caracteres se ha utilizado la técnica conocida como análisis de correspondencias óptimas (optimal matching analysis), que permite reconocer individuos con recorridos similares a lo largo del tiempo en una serie de identificadores categóricos. De esta forma se pueden realizar exámenes de conglomerados como los que se plantean en este trabajo, relacionando los itinerarios educativos que se asemejen entre ellos y a su vez más se diferencien de los otros.

Las secuencias identificadas por cada individuo pueden ser agrupadas mediante el procedimiento de análisis de aglomeración (análisis clúster) siguiendo la metodología de análisis de correspondencias óptimas. Mediante esta técnica se calculan las distancias entre las diferentes secuencias de los individuos, y posteriormente se genera una matriz que permite agrupar los casos similares. El criterio que se ha utilizado para llevar a cabo la agrupación jerárquica es el método Ward de varianza mínima. Este método permite medir la pérdida de información que se produce al integrar a los distintos individuos en agrupaciones mediante la suma total de los cuadrados de las desviaciones entre cada punto (individuo) y la media del conglomerado en el que se integra (Ward, 1963).

\subsection{Características de las trayectorias: variables de análisis}

En los diferentes modelos de análisis de regresión logística multinomial, se seleccionan como variables endógenas los conglomerados de las trayecto- 
rias resultantes del análisis clúster. A partir de las hipótesis establecidas se identifican cinco factores explicativos relacionados con las trayectorias educativas de los jóvenes. El primero es el sexo («hombre» como categoría de referencia). El segundo, el origen del estudiante, dividido en dos categorías: "autóctono», como categoría de referencia, e «inmigrante», o nacido fuera de España. En tercer lugar, el nivel educativo de los padres se divide en tres categorías: que el total de los progenitores tengan como máximo estudios finalizados de "primaria o inferiores" (categoría referencia), que alguno de ellos tenga como máximo estudios «secundarios» finalizados y que alguno tenga estudios «superiores». En cuarto lugar, el apoyo parental recibido se mide en dos categorías, "apoyo medio-alto" y "apoyo bajo-nulo" (categoría de referencia), a partir de la pregunta "¿Tus padres te han animado, en general, a continuar estudiando?», con cuatro categorías de respuesta: «mucho», "bastante», "poco» y «nada». Por último, el apoyo recibido por tutores o maestros también se incluye en los modelos de análisis a partir de una pregunta con el mismo formato que la anterior ("¿ Tus maestros y tutores te han animado, en general, a continuar estudiando?»), con la misma categorización de respuestas («apoyo bajo-nulo» como categoría de referencia).

\section{Resultados}

\subsection{Trayectorias educativas de los jóvenes catalanes a partir del análisis de secuencias}

La EJC12 recoge once situaciones diferentes en las que los jóvenes pueden encontrarse en relación con el sistema educativo catalán:

1. Escuela taller, Plan de Garantía Social (PGS), Programas de Cualificación Profesional Inicial (PCPI), escuela de adultos, otros similares (excluida la formación ocupacional durante el paro).

2. Educación obligatoria (ESO o EGB).

3. BUP, COU o bachillerato.

4. Formación profesional nivel 1 (cursos de formación de grado medio).

5. Formación profesional nivel 2 (cursos de formación de grado superior).

6. Diplomatura o equivalente (incluye grados actuales).

7. Licenciatura (incluye enseñanzas artísticas de regímenes especiales) o equivalente (incluye grados actuales).

8. Postgrado o máster.

9. Programas que requieren una titulación universitaria (MIC, CAP, FIR, BIP). 10. Doctorado.

11. No estudiando.

1. La categoría de apoyo parental medio-alto es la suma de las dos primeras categorías de respuesta, y la categoría de apoyo bajo-nulo, la suma de las dos últimas. 
Para operativizar las categorías a fin de realizar el análisis de secuencias, se han agrupado los niveles educativos en grados aproximados o equivalentes. Las categorías resultantes son seis:

a) No estudiando (NE).

b) Graduado escolar (GE): educación obligatoria (ESO o EGB) + escuelas taller, PGS, PCPI, escuela de adultos y otros similares.

c) Estudios de secundaria postobligatoria (SPO): BUP, COU o bachillerato + FP1 / CF grado medio.

d) Graduado superior (GS): FP2 / CF grado superior.

e) Diplomaturas, licenciaturas o grados (DLG): diplomatura o equivalente + licenciatura o equivalente (incluye grados actuales).

f) Postgrado, máster o doctorado (PMD): postgrado o máster + programas que requieren una titulación universitaria + doctorado.

En el gráfico 1 se pueden observar las trayectorias educativas de los jóvenes según las categorías previamente definidas. El ancho de cada categoría dentro de la columna indica el peso relativo de cada condición en un determinado tramo de edad (cuatrimestral). Según estos resultados, a los 15 años en torno al 4,5\% de la muestra de jóvenes (la mayoría inmigrantes) ya no estaban estudiando a pesar de que la enseñanza obligatoria en España es hasta los 16 años. Esta categoría alcanza el 50\% entre los 20 y los 21 años. Es en estas edades cuando se llega al punto álgido de la proporción de estudiantes que cursan algún grado universitario. A partir de los 23 años se va reduciendo progresivamente la proporción de alumnos en todas las categorías (menos en

Gráfico 1. Trayectorias educativas de los jóvenes de 15 a 30 años

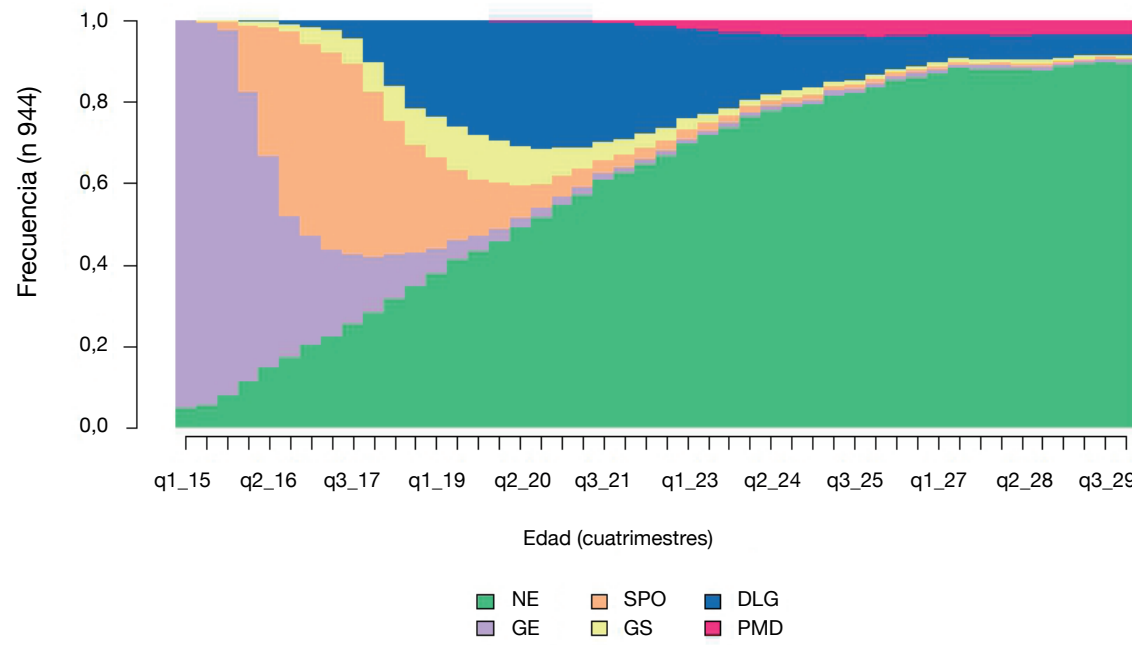

Fuente: elaboración propia a partir de EJC12. 
Tabla 1. Análisis de entropía longitudinal. Relación de los niveles educativos con el sexo, el origen y los niveles educativos de los padres en los jóvenes de 15 a 30 años

\begin{tabular}{|c|c|c|}
\hline Variables exógenas & Coeficientes & Error estándar \\
\hline \multicolumn{3}{|l|}{ Sexo (ref. hombre) } \\
\hline Mujer & $0,073^{\star \star \star}$ & 0,014 \\
\hline \multicolumn{3}{|l|}{ Origen (ref. inmigrante) } \\
\hline Autóctono & $0,149^{\star \star \star}$ & 0,017 \\
\hline \multicolumn{3}{|c|}{ Nivel de estudios de los padres (ref. superiores) } \\
\hline Obligatorios o inferiores & $-0,163^{\star \star \star}$ & 0,017 \\
\hline Secundarios postobligatorios & $-0,053^{\star \star}$ & 0,019 \\
\hline (Constante) & $0,423^{\star \star \star}$ & 0,020 \\
\hline $\mathrm{R}^{2}$ Ajustada & 0,17 & \\
\hline Número de casos & 944 & \\
\hline Nivel de significación: ${ }^{*} p<0,05 ;{ }^{* *} p<0,0$ & & \\
\hline
\end{tabular}

Fuente: elaboración propia a partir de EJC12.

los niveles superiores universitarios), pero se mantienen constantes a partir de los 27 años de edad.

Estas trayectorias esconden variaciones significativas en los itinerarios en función de variables sociodemográficas como es el caso del género. Según el análisis de las trayectorias de los chicos y de las chicas por separado, entre los 15 y 30 años los chicos pasan más tiempo de promedio sin estar estudiando que las chicas. Del mismo modo, hay una mayor proporción de chicas en niveles universitarios, especialmente desde los 18 años hasta los 26 años aproximadamente (a partir de esta edad, las diferencias son reducidas). La mujeres entre los 15 y los 30 años transcurren una media de 6 años y medio fuera del sistema educativo ( 26 cuatrimestres), mientras que en el caso de los varones se trata de 7 años (28 cuatrimestres). Mediante el análisis de entropía longitudinal (ver Gabadinho et al., 2011), se puede examinar en qué medida la diversidad de estados entre cada secuencia está relacionada con el sexo de los jóvenes (controlado por el origen y el nivel educativo de los padres). Los resultados muestran que las mujeres y los estudiantes nacidos en España experimentan mayor diversidad de estados (tabla 1). Según cálculos realizados, los chicos estarían un promedio de 9 años y 4 meses fuera de sistema educativo entre los 15 y los 30 años, en cambio las chicas estarían un año menos de promedio en el mismo periodo de edad. Por otro lado, los estudiantes que tienen padres con niveles de estudios más bajos a los estudios superiores tienden a transitar por menos etapas educativas.

\subsection{Tipología de las trayectorias de los jóvenes y factores asociados}

Para seleccionar el número de conglomerados óptimo de las trayectorias educativas de los jóvenes, hemos utilizado tanto parámetros estadísticos como teóricos. Según el análisis de la anchura de la silueta (ver Rousseeuw, 1987), los dos 
Gráfico 2. Trayectorias educativas de los jóvenes de 15 a 30 años según agrupaciones similares (clústeres)
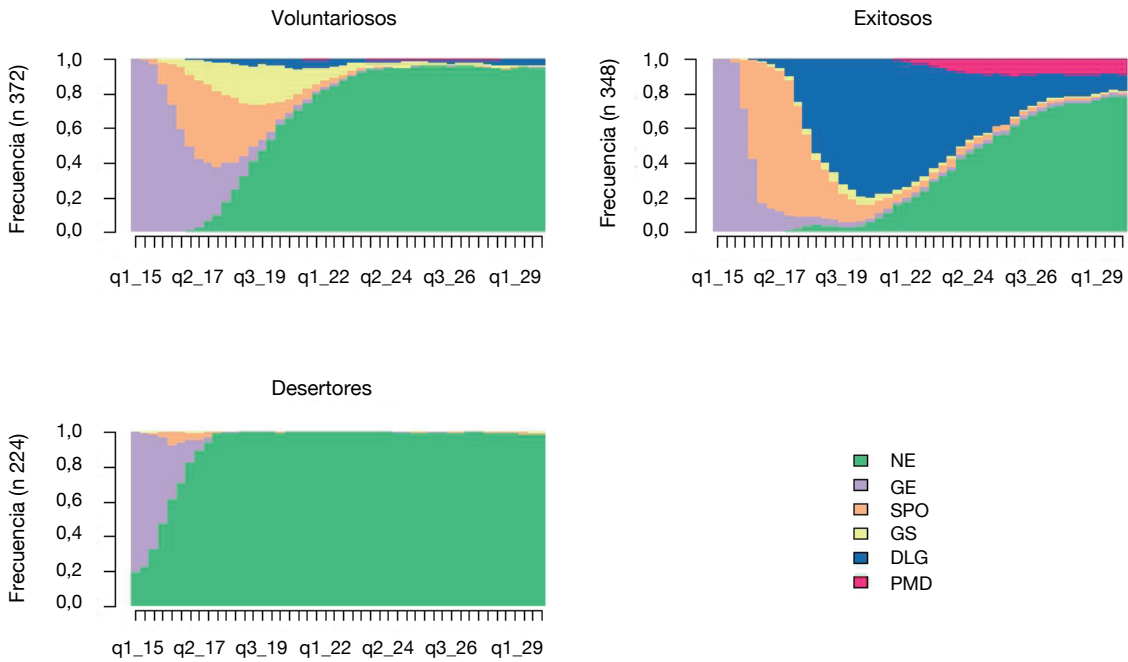

Fuente: elaboración propia a partir de EJC12.

modelos más convenientes serían los formados por dos agrupaciones (anchura de la silueta $=0,5$ ) y por tres agrupaciones (anchura de la silueta $=0,4$ ). Mientras que en la tipología de dos clústeres el promedio en que están los jóvenes fuera del sistema educativo de los 15 a los 30 años en el primer conglomerado es de aproximadamente 15 cuatrimestres, y en el segundo, de 35 cuatrimestres (gráfico A.1, en apéndice), en la tipología de tres clústeres obtenemos una agrupación con un promedio aproximado de 15 (la misma que en el caso de la tipología de dos clústeres), otra de 30 y la última de 43 cuatrimestres (gráfico A.2, en apéndice). En este sentido parece razonable plantear un análisis separando las trayectorias de este último grupo de jóvenes que escasamente se mantienen en el sistema educativo a partir de los 15 años (un 23,7\% de la muestra), y que en ningún caso llegan a la educación postobligatoria, de aquellos que permanecen significativamente más tiempo en él. En definitiva, a partir de las trayectorias de los jóvenes catalanes, la tipología de clústeres formada por tres conjuntos de itinerarios resulta ser la más óptima teniendo en cuenta los indicadores estadísticos y los criterios teóricos. Estos tres conglomerados de trayectorias mantienen diferencias significativas (gráfico 2).

La primera agrupación (representa el 39,4\% de la muestra) se compone de estudiantes que permanecen más tiempo realizando el graduado escolar, y en torno al $60 \%$ cursan en algún momento estudios secundarios postobligatorios o ciclos formativos de grados superiores. También hay un porcentaje, aunque reducido (entorno al 5\%), de alumnos que realizan en algún momento estudios universitarios en este tipo de trayectoria. Definimos esta agrupación de trayectorias como la formada por jóvenes «voluntariosos». 
Tabla 2. Características de los jóvenes en las agrupaciones de trayectorias educativas (\%)

\begin{tabular}{lccc}
\hline & Voluntariosos & Exitosos & Desertores \\
\hline Sexo & & & \\
$\quad$ Hombre & 49,0 & 45,8 & 66,8 \\
$\quad$ Mujer & 51,0 & 54,2 & 33,2 \\
\hline Origen & & & \\
$\quad$ Autóctono & 75,5 & 85,1 & 70,2 \\
$\quad$ Nacido fuera de España & 24,5 & 14,9 & 29,8 \\
\hline Nivel de estudios de los padres & & & \\
$\quad$ Primarios o inferiores & 51,1 & 31,8 & 68,5 \\
Secundarios & 27,6 & 30,0 & 24,0 \\
$\quad$ Superiores & 21,2 & 38,2 & 7,4 \\
\hline Apoyo parental & & & \\
$\quad$ Apoyo medio-alto & 78,8 & 93,1 & 67,8 \\
$\quad$ Apoyo bajo-nulo & 21,2 & 6,9 & 32,2 \\
\hline Apoyo de los tutores y/o de los maestros & & & \\
Apoyo medio-alto & 64,7 & 79,9 & 53,0 \\
Apoyo bajo-nulo & 35,3 & 20,1 & 47,0 \\
\hline
\end{tabular}

Fuente: elaboración propia a partir de EJC12.

Tienen una mayor proporción de individuos que llegan a seguir estudios de grado superior (FP2/CFGS), que pasan más tiempo de media en el sistema educativo que el segundo clúster y menos que el tercero. La segunda agrupación (el 36,9\% de la muestra) está formada, en su mayoría, por jóvenes que permanecen significativamente más tiempo de promedio en el sistema educativo. Un mayor porcentaje de ellos llega a cursar estudios universitarios y superiores tras haber cursado la secundaria postobligatoria. Son los «exitosos» educativos. Y, por último, el tercer grupo (contiene el 23,7\% restante de jóvenes) está formado principalmente por los jóvenes que abandonan el sistema educativo de forma más temprana. A este grupo los llamamos los «desertores» educativos.

A modo de síntesis, las trayectorias medoides o centroides de cada agrupación -que representarían la centralidad de cada conglomerado mediante la selección del individuo «típico» o central- muestran las evidentes diferencias entre cada agrupación de itinerarios (cada paréntesis está formado por la situación del individuo en relación con el sistema educativo acompañado del número de cuatrimestres promedio). Así, la trayectoria que representa la centralidad en las diferentes trayectorias que componen los voluntariosos sería la siguiente: $(\mathrm{GE}-8)-(\mathrm{SPO}-6)-(\mathrm{NE}-32)$; para los exitosos: $(\mathrm{GE}-5)-(\mathrm{SPO}-6)-$ $(\mathrm{DLG}-17)-(\mathrm{NE}-18)$, y para los desertores: $(\mathrm{GE}-4)-(\mathrm{NE}-42)$.

En la tabla 2 se presentan los porcentajes de jóvenes distribuidos en las diferentes agrupaciones de trayectorias educativas según las variables y las categorías establecidas previamente para el análisis de las regresiones logísticas multinomiales. 
Tabla 3. Modelos de regresión logit multinomial. Razón de probabilidades (odds ratio) de pertenecer a alguna de las agrupaciones de trayectorias educativas de los jóvenes

\begin{tabular}{|c|c|c|c|c|c|c|}
\hline \multirow{2}{*}{ Sexo (ref. hombre) } & \multicolumn{2}{|c|}{ Exit.-vol. (ref.) } & \multicolumn{2}{|c|}{ Des.-vol. (ref.) } & \multicolumn{2}{|c|}{ Des.-exit. (ref.) } \\
\hline & & & & & & \\
\hline Mujer & 1,13 & $(0,16)$ & $0,470^{\star \star \star}$ & $(0,19)$ & $0,416^{\star \star *}$ & $(0,21)$ \\
\hline \multicolumn{7}{|l|}{ Origen (ref. autóctono) } \\
\hline Inmigrante & $0,381^{\star * *}$ & $(0,21)$ & $1,534^{*}$ & $(0,22)$ & $4,030^{\star \star \star}$ & $(0,26)$ \\
\hline \multicolumn{7}{|l|}{$\begin{array}{l}\text { Nivel de estudios de los padres } \\
\text { (ref. primaria o inferiores) }\end{array}$} \\
\hline Secundarios & $1,789^{\star \star}$ & $(0,19)$ & $0,621^{*}$ & $(0,22)$ & $0,347^{\star \star \star}$ & $(0,23)$ \\
\hline Superiores & $2,979^{\star \star \star}$ & $(0,20)$ & $0,274^{\star \star \star}$ & $(0,31)$ & $0,092^{\star \star \star}$ & $(0,32)$ \\
\hline \multicolumn{7}{|l|}{ Apoyo parental (ref. apoyo bajo-nulo) } \\
\hline Apoyo medio-alto & $3,047^{\star \star \star}$ & $(0,28)$ & 0,693 & $(0,22)$ & $0,227^{\star \star \star}$ & $(0,30)$ \\
\hline \multicolumn{7}{|l|}{$\begin{array}{l}\text { Apoyo de los tutores y/o de los maestros } \\
\text { (ref. apoyo bajo-nulo) }\end{array}$} \\
\hline Apoyo medio-alto & $1,756^{\star \star}$ & $(0,19)$ & 0,809 & $(0,20)$ & $0,461^{\star \star \star}$ & $(0,23)$ \\
\hline Constante (coeficiente B no estandarizado) & $-1,80^{\star \star \star}$ & & 0,314 & & $2,110^{\star \star \star}$ & \\
\hline$N$ & 944 & & 944 & & 944 & \\
\hline Nagelkerke pseudo R cuadrada & 0,244 & & 0,244 & & 0,244 & \\
\hline-2 log likelihood (final) & 352,3 & & 352,3 & & 352,3 & \\
\hline
\end{tabular}

Leyenda: coeficientes y errores estándar entre paréntesis. Nivel de significación: ${ }^{*} p<0,05,{ }^{* *} p<0,01$ y ${ }^{* * \star} p<0,001$.

Nota: la abreviatura ref. indica la categoría de referencia. La abreviatura vol. significa 'voluntariosos'; exit., 'exitosos', y des., 'desertores'.

Fuente: elaboración propia a partir de la EJC12.

A partir de las variables seleccionadas elaboramos diferentes regresiones logísticas multinomiales que permiten identificar la asociación de las variables con los tipos de trayectorias educativas de los jóvenes. En la tabla 3 las odds ratio $(\mathrm{OR})$ indican en qué medida la pertenencia a una determinada aglomeración se asocia a ciertas condiciones en comparación con los otros conglomerados de trayectorias. Según estos resultados, la razón de probabilidad de seguir el patrón de la trayectoria de los exitosos es 2,4 veces mayor para las mujeres que para los hombres en comparación con los desertores $(p<0,001)$. A su vez, la probabilidad de seguir el modelo de trayectoria de los voluntariosos en comparación con los desertores es 2,1 veces mayor en las mujeres que en los hombres $(p<0,001)$. Así pues, se confirma suficientemente la $\mathrm{H} 1$ a pesar de que, en el modelo de trayectoria de los voluntariosos en comparación con los exitosos, los resultados no son estadísticamente significativos según la variable sexo.

En el caso de los inmigrantes, tienen una probabilidad cuatro veces mayor de situarse en las trayectorias de desertores que los autóctonos en comparación con los exitosos $(p<0,001), 1,5$ veces mayor en trayectorias de tipo desertor en referencia a los voluntariosos $(p<0,05)$ y 2,6 veces menor de situarse en itinerarios educativos voluntariosos que exitosos $(p<0,001)$. Estos 
resultados están en sintonía con los obtenidos en los análisis de Feliciano y Rumbaut (2005), que señalaban que las mujeres inmigrantes desarrollaban mayores expectativas académicas, lo que redundaba en trayectorias educativas más continuas. El nivel de estudios de los padres ejerce una fuerte influencia, especialmente cuando se comparan los niveles más extremos, tal y como sugieren los análisis realizados por Milesi (2010). Los estudiantes con padres de niveles educativos superiores tienen menos probabilidad de pertenecer a las trayectorias de los desertores y más probabilidad de ubicarse en trayectorias exitosas. Concretamente, 10,9 veces mayor probabilidad de no pertenecer al patrón de la trayectoria de los desertores $(p<0,001)$ y 3 veces de no pertenecer a los voluntariosos $(p<0,001)$ en comparación con los exitosos y respecto a los estudiantes que tienen padres con estudios de niveles bajos. También supone que los voluntariosos tengan una mayor probabilidad de ubicarse en el patrón de trayectorias educativas que de los desertores.

Según los resultados, los estudiantes que han recibido un apoyo parental medio-alto tienen 4,4 más probabilidades de seguir una trayectoria educativa exitosa en comparación con las trayectorias desertoras $(p<0,001)$ y 3 veces mayor probabilidad en comparación con las voluntariosas $(p<0,01)$ respecto a los estudiantes que no reciben este nivel de apoyo parental. Respecto a la influencia que ejercen los referentes educativos o académicos en la motivación de los alumnos para continuar con los estudios, vemos que el hecho de recibir un apoyo medio-alto también ejerce una influencia significativa, pero con un efecto más reducido que en el caso de las figuras parentales. La OR en las trayectorias exitosas es 2,2 veces mayor para los que reciben un apoyo medio-alto respecto a los que reciben un apoyo escaso o nulo por parte de los profesores $(p<0,001)$ en comparación con las trayectorias de desertores, y 1,8 menor en el caso de los voluntariosos en comparación con los exitosos $(p<0,01)$.

Los modelos que muestran las tablas 4, 5 y 6 contienen la misma estructura de variables con el añadido del efecto interactivo entre el sexo y el resto de variables explicativas establecidas (una por modelo). De esta forma se captura el efecto que el género pueda ejercer sobre el resto de las variables minimizando o aumentando la probabilidad de pertenecer a uno u otro itinerario educativo en comparación con el resto. El primer modelo de las tres tablas muestra el efecto de la interacción entre el sexo y el origen de los jóvenes. De las tres categorías de itinerarios educativos cabe destacar el efecto que tiene esta interacción en el modelo de las trayectorias de los desertores: En la tabla 6 modelo Mc1, la condición de mujer aumenta la OR de pertenecer a la trayectoria educativa exitosa en comparación con la desertora $(\mathrm{OR}=0,22 ; p<0,001)$. Este efecto, no obstante, parece ser más reductor para los autóctonos que para los inmigrantes, ya que en las mujeres de origen inmigrante se produce un ajuste al alza de OR de pertenecer a la trayectoria educativa de desertores de 10,5 ( $p<$ $0,001)$ en comparación con los exitosos. En el mismo sentido, los resultados muestran un aumento de las probabilidades de pertenecer a las trayectorias educativas desertoras en comparación con las voluntariosas cuando las mujeres 
Tabla 4. Modelos de regresión logit multinomial. Razón de probabilidades (odds ratio) de pertenecer a las trayectorias educativas de exitosos comparadas con las de voluntariosos (ref.)

\begin{tabular}{|c|c|c|c|c|c|c|c|c|}
\hline \multirow{2}{*}{ Sexo (ref. hombre) } & \multicolumn{2}{|c|}{ Ma1 } & \multicolumn{2}{|c|}{ Ma2 } & \multicolumn{2}{|c|}{ Ma3 } & \multicolumn{2}{|c|}{ Ma4 } \\
\hline & & & & & & & & \\
\hline Mujer & 1,093 & $(0,18)$ & $0,553^{*}$ & $(0,26)$ & 0,749 & $(0,53)$ & 0,773 & $(0,32)$ \\
\hline \multicolumn{9}{|l|}{ Origen (ref. autóctono) } \\
\hline Inmigrante & $0,413^{\star \star}$ & $(0,31)$ & $0,375^{\star \star \star}$ & $(0,22)$ & $0,379^{\star \star \star}$ & $(0,21)$ & $0,376^{\star \star \star}$ & $(0,22)$ \\
\hline \multicolumn{9}{|l|}{$\begin{array}{l}\text { Nivel de estudios de los padres } \\
\text { (ref. primaria o inferiores) }\end{array}$} \\
\hline Secundarios & $1,809^{\star \star}$ & $(0,19)$ & 0,854 & $(0,28)$ & $1,786^{\star \star}$ & $(0,19)$ & $1,818^{\star \star}$ & $(0,19)$ \\
\hline Superiores & $3,003^{\star \star *}$ & $(0,20)$ & $2,125^{\star \star}$ & $(0,29)$ & $2,980^{\star \star \star}$ & $(0,20)$ & $3,022^{\star \star \star}$ & $(0,20)$ \\
\hline \multicolumn{9}{|l|}{ Apoyo parental (ref. apoyo bajo-nulo) } \\
\hline Apoyo medio-alto & $3,030^{\star \star *}$ & $(0,28)$ & $2,996^{\star \star \star}$ & $(0,28)$ & $2,585^{\star *}$ & $(0,35)$ & $2,904^{\star \star *}$ & $(0,28)$ \\
\hline \multicolumn{9}{|l|}{$\begin{array}{l}\text { Apoyo de los tutores y/o de los maestros } \\
\text { (ref. apoyo bajo-nulo) }\end{array}$} \\
\hline Apoyo medio-alto & $1,745^{\star \star}$ & $(0,19)$ & $1,862^{\star \star}$ & $(0,19)$ & $1,724^{\star \star}$ & $(0,19)$ & 1,376 & $(0,26)$ \\
\hline \multicolumn{9}{|l|}{ Interacción entre el sexo* y el origen } \\
\hline Mujer* inmigrante & 0,905 & $(0,42)$ & & & & & & \\
\hline \multicolumn{9}{|l|}{$\begin{array}{l}\text { Interacción entre el sexo* y el nivel } \\
\text { de estudios de los padres }\end{array}$} \\
\hline Mujer* secundarios postobligatorios & & & $4,434^{\star \star \star}$ & $(0,40)$ & & & & \\
\hline Mujer* superiores & & & 2,081 & $(0,40)$ & & & & \\
\hline
\end{tabular}

Interacción entre el sexo*

y el apoyo parental

Mujer* apoyo medio-alto $1,517 \quad(0,56)$

Interacción entre el sexo* y el apoyo

de los tutores y/o de los maestros

\begin{tabular}{|c|c|c|c|c|}
\hline Mujer apoyo medio-alto & & & & 1,674 \\
\hline $\begin{array}{l}\text { Constante (coeficiente B } \\
\text { no estandarizado) }\end{array}$ & $-1,78^{\star \star \star}$ & $-1,50^{\star \star}$ & $-1,62^{\star \star \star}$ & $-1,59^{\star \star \star}$ \\
\hline 1 & 944 & 944 & 944 & 944 \\
\hline Nagelkerke pseudo $R$ cuadrada & 0,273 & 0,278 & 0,249 & 0,246 \\
\hline-2 log likelihood (final) & 322,6 & 317,7 & 347,6 & 350,4 \\
\hline
\end{tabular}

Leyenda: coeficientes y errores estándar entre paréntesis. Nivel de significación: ${ }^{*} p<0,05,{ }^{* *} p<0,01$ y ${ }^{* \star *} p<0,001$.

Nota: la abreviatura ref. indica la categoría de referencia.

Fuente: elaboración propia a partir de la EJC12.

son inmigrantes (OR: 9,5; $p<0,001$; tabla 5, modelo Mb1). Estos resultados avalarían la $\mathrm{H} 2$ y van más allá de los obtenidos en investigaciones tales como las de Feliciano y Rumbaut (2005), que realizan comparativas de rendimientos y expectativas educativas entre hombres y mujeres inmigrantes sin compararlos con los autóctonos. Este tipo de estudios, al no confrontar sus resultados con los autóctonos, pierden cierta riqueza analítica, ya que, como muestran las 
Tabla 5. Modelos de regresión logit multinomial. Razón de probabilidades (odds ratio) de pertenecer a las trayectorias educativas de desertores comparadas con las de voluntariosos (ref.)

\begin{tabular}{|c|c|c|c|c|c|c|c|c|}
\hline Sexo (ref. hombre) & \multicolumn{2}{|c|}{ Mb1 } & \multicolumn{2}{|c|}{$\mathrm{Mb2}$} & \multicolumn{2}{|c|}{ Mb3 } & \multicolumn{2}{|c|}{ Mb4 } \\
\hline Mujer & $0,239^{\star \star \star}$ & $(0,25)$ & 0,644 & $(0,23)$ & 0,789 & $(0,35)$ & $0,413^{\star \star}$ & $(0,30)$ \\
\hline \multicolumn{9}{|l|}{ Origen (ref. autóctono) } \\
\hline Inmigrante & $0,524^{*}$ & $(0,32)$ & 1,516 & $(0,22)$ & $1,592^{*}$ & $(0,22)$ & 1,522 & $(0,22)$ \\
\hline \multicolumn{9}{|l|}{$\begin{array}{l}\text { Nivel de estudios de los padres } \\
\text { (ref. primaria o inferiores) }\end{array}$} \\
\hline Secundarios & 0,656 & $(0,22)$ & 0,785 & $(0,27)$ & $0,622^{*}$ & $(0,22)$ & $0,623^{\star}$ & $(0,22)$ \\
\hline Superiores & $0,280^{\star \star \star}$ & $(0,32)$ & $0,475^{\star}$ & $(0,37)$ & $0,273^{\star \star \star}$ & $(0,31)$ & $0,277^{\star \star \star}$ & $(0,31)$ \\
\hline \multicolumn{9}{|l|}{ Apoyo parental (ref. apoyo bajo-nulo) } \\
\hline Apoyo medio-alto & $0,600^{*}$ & $(0,23)$ & 0,706 & $(0,22)$ & 0,913 & $(0,28)$ & 0,687 & $(0,22)$ \\
\hline \multicolumn{9}{|l|}{$\begin{array}{l}\text { Apoyo de los tutores y/o de } \\
\text { los maestros (ref. apoyo bajo-nulo) }\end{array}$} \\
\hline Apoyo medio-alto & 0,785 & $(0,20)$ & 0,768 & $(0,20)$ & 0,355 & $(0,20)$ & 0,733 & $(0,25)$ \\
\hline \multicolumn{9}{|l|}{ Interacción entre el sexo* y el origen } \\
\hline Mujer* inmigrante & $9,496^{\star \star \star}$ & $(0,45)$ & & & & & & \\
\hline \multicolumn{9}{|l|}{$\begin{array}{l}\text { Interacción entre el sexo* y el nivel } \\
\text { de estudios de los padres }\end{array}$} \\
\hline Mujer* secundarios postobligatorios & & & $0,369^{\star}$ & $(0,51)$ & & & & \\
\hline Mujer* superiores & & & $0,097^{\star}$ & $(1,02)$ & & & & \\
\hline
\end{tabular}

Interacción entre el sexo* y el apoyo parental

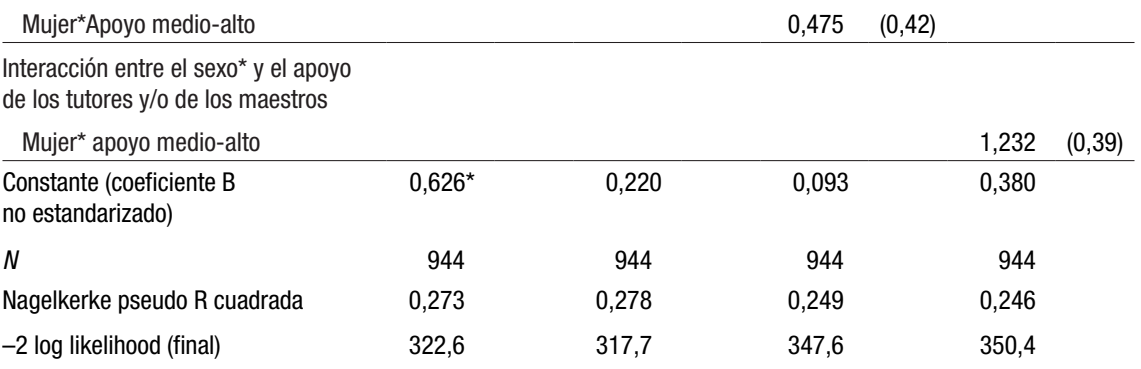

Leyenda: coeficientes y errores estándar entre paréntesis. Nivel de significación: ${ }^{*} p<0,05 ;{ }^{* *} p<0,01$; ${ }^{* * *} p<0,001$.

Nota: la abreviatura ref. indica la categoría de referencia.

Fuente: elaboración propia a partir de la EJC12.

conclusiones de la presente investigación, las desigualdades de género están significativamente diferenciadas según el origen.

El modelo 2 de cada tabla mide el impacto de la interacción entre los niveles educativos de los padres y el sexo de los estudiantes. En este sentido hay que destacar los modelos Mb2 (tabla 5) y Mc2 (tabla 6). Los datos muestran que tener padres con niveles académicos que van más allá de los primarios 
Tabla 6. Modelos de regresión logit multinomial. Razón de probabilidades (odds ratio) de pertenecer a las trayectorias educativas de desertores comparadas con las de exitosos (ref.)

\begin{tabular}{|c|c|c|c|c|c|c|c|c|}
\hline \multirow{2}{*}{ Sexo (ref. hombre) } & \multicolumn{2}{|c|}{ Mc1 } & \multicolumn{2}{|c|}{ Mc2 } & \multicolumn{2}{|c|}{ Mc3 } & \multicolumn{2}{|c|}{ Mc4 } \\
\hline & & & & & & & & \\
\hline Mujer & $0,219^{\star \star \star}$ & $(0,26)$ & 1,165 & $(0,28)$ & 1,054 & $(0,56)$ & 0,534 & $(0,37)$ \\
\hline \multicolumn{9}{|l|}{ Origen (ref. autóctono) } \\
\hline Inmigrante & 1,267 & $(0,38)$ & $4,042^{\star \star \star}$ & $(0,26)$ & $4,200^{\star \star \star}$ & $(0,26)$ & $4,053^{\star \star \star}$ & $(0,26)$ \\
\hline \multicolumn{9}{|l|}{$\begin{array}{l}\text { Nivel de estudios de los padres } \\
\text { (ref. primaria o inferiores) }\end{array}$} \\
\hline Secundarios & $0,363^{\star \star \star}$ & $(0,24)$ & 0,919 & $(0,30)$ & $0,348^{\star \star \star}$ & $(0,23)$ & $0,345^{\star \star \star}$ & $(0,23)$ \\
\hline Superiores & $0,093^{\star \star \star}$ & $(0,32)$ & $0,223^{\star \star \star}$ & $(0,36)$ & $0,092^{\star \star \star}$ & $(0,32)$ & $0,092^{\star \star \star}$ & $(0,32)$ \\
\hline \multicolumn{9}{|l|}{ Apoyo parental (ref. apoyo bajo-nulo) } \\
\hline Apoyo medio-alto & $0,198^{\star \star \star}$ & $(0,30)$ & $0,236^{\star \star \star}$ & $(0,30)$ & $0,353^{\star \star}$ & $(0,37)$ & $0,237^{\star \star \star}$ & $(0,30)$ \\
\hline \multicolumn{9}{|l|}{$\begin{array}{l}\text { Apoyo de los tutores y/o de } \\
\text { los maestros (ref. apoyo bajo-nulo) }\end{array}$} \\
\hline Apoyo medio-alto & $0,450^{\star \star \star}$ & $(0,23)$ & $0,413^{\star \star \star}$ & $(0,23)$ & $0,481^{\star \star}$ & $(0,23)$ & $0,533^{*}$ & $(0,28)$ \\
\hline \multicolumn{9}{|l|}{ Interacción entre el sexo* y el origen } \\
\hline Mujer* inmigrante & $10,494^{\star \star \star}$ & $(0,53)$ & & & & & & \\
\hline \multicolumn{9}{|l|}{$\begin{array}{l}\text { Interacción entre el sexo* y el nivel } \\
\text { de estudios de los padres }\end{array}$} \\
\hline Mujer* secundarios postobligatorios & & & $0,083^{\star \star \star}$ & $(0,54)$ & & & & \\
\hline Mujer* superiores & & & $0,047^{\star \star}$ & $(1,02)$ & & & & \\
\hline
\end{tabular}

Interacción entre el sexo* $y$ el apoyo

parental

Mujer* apoyo medio-alto $0,313 \quad(0,61)$

Interacción entre el sexo* y el apoyo

de los tutores y/o de los maestros

\begin{tabular}{lrrrrr}
\multicolumn{1}{l}{ Mujer* apoyo medio-alto } & & & 0,736 & $(0,45)$ \\
\hline Constante (coeficiente B & $2,407^{\star \star \star}$ & $1,717^{\star \star \star}$ & $1,712^{\star \star \star}$ & $1,964^{\star \star \star}$ \\
no estandarizado) & & & & \\
$N$ & 944 & 944 & 944 & 944 \\
$N$ Nagelkerke pseudo R cuadrada & 0,273 & 0,278 & 0,249 & 0,246 \\
-2 log likelihood (final) & 322,6 & 317,7 & 347,6 & 350,4 & \\
\hline
\end{tabular}

Leyenda: coeficientes y errores estándar entre paréntesis. Nivel de significación: ${ }^{*} p<0,05,{ }^{* *} p<0,01$ y ${ }^{* * \star} p<0,001$

Nota: la abreviatura ref. indica la categoría de referencia.

Fuente: elaboración propia a partir de la EJC12.

tiene un efecto reductor de la probabilidad de que los jóvenes acaben teniendo itinerarios educativos de abandono temprano, especialmente entre las mujeres. Se produce un ajuste a la baja de $\mathrm{OR}=0,083(p<0,001)$ en el supuesto de tener padres con estudios secundarios y de OR $=0,047(p<0,01)$ en el de tener padres con niveles educativos superiores, lo que implica una reducción de hasta 21,3 veces el efecto de las chicas respeto a los chicos con padres con 
niveles educativos bajos de ubicarse en las trayectorias de los desertores en comparación con los exitosos (tabla 6, modelo Mc2). En otras palabras, el efecto reductor que tiene el nivel educativo de los padres parece ser mayor en las jóvenes estudiantes que entre sus iguales masculinos. En la misma línea encontramos efectos estadísticamente significativos pero de menor intensidad en la tabla 5, donde se observa que el impacto positivo que se obtiene de tener padres con estudios secundarios o superiores para transitar por un itinerario de voluntariosos es superior en el caso de las mujeres. Los resultados de la interacción entre los niveles educativos de los padres y el género en las trayectorias se presumen diferenciados según los niveles educativos de cada miembro, distinguiendo los de la madre y los del padre, como apuntan algunos estudios (Sewell y Shah, 1968; Kim et al., 2015).

Respecto al tercer modelo, el que mide el efecto de la interacción entre el apoyo parental y el sexo de los jóvenes, no encontramos significatividad estadística en ninguna de las comparativas entre modelos. Así pues, no vemos evidencias que confirmen la H4. A su vez, tampoco encontramos significatividad en todos los modelos cuando incluimos la interacción entre el apoyo del referente educativo (tutores o maestros) y el sexo del estudiante (H5). Es importante indicar que los resultados no son del todo concluyentes respecto a la diferencia de género, debido a que en los análisis no se ha podido distinguir si el apoyo provenía de la madre o del padre ni tampoco del profesor o de la profesora.

\section{Conclusión}

En este trabajo se ha presentado una tipología innovadora de las trayectorias educativas de los jóvenes en Cataluña mediante el análisis de secuencias aplicable al resto de comunidades autónomas o en España. Ampliar el conocimiento en determinar qué trayectorias son las que hay que potenciar y cuáles son los factores que se asocian con diferentes itinerarios educativos es clave para tratar de plantear medidas que busquen reducir las trayectorias menos funcionales para los jóvenes y la sociedad. En los últimos años, los datos han mostrado cómo en Cataluña (así como en España) se mantienen indicadores educativos (como el logro académico o el abandono prematuro escolar) en niveles deficientes en comparación con la mayoría de países de nuestro entorno. Estos datos están significativamente diferenciados por géneros, puesto que mantienen una brecha constante y creciente que posiciona a los estudiantes varones en una peor situación que a las chicas. En este sentido, dicha investigación ha permitido dar un paso más en la tarea de identificar la tipología de trayectorias que determinan los estudiantes, la magnitud de cada una y cuales están asociadas al género.

Prácticamente todos los factores analizados influyen sobre las trayectorias educativas. El efecto de estos varía tanto según el sexo como también según el itinerario analizado. El efecto reductor que tiene ser mujer en la probabilidad de pertenecer a un itinerario con un alto grado de abandono educativo temprano se produce en mayor medida para los autóctonos, mientras que en 
los inmigrantes el efecto de género sería menor. Los datos muestran que, en el análisis de las trayectorias educativas entre hombres y mujeres, es particularmente relevante tener en cuenta la diferenciación según el origen, ya que las pautas cambian significativamente. Otra conclusión que se obtiene del análisis diferenciado por géneros es la importancia del nivel académico de los padres, no solo como determinante del logro educativo, sino también de los itinerarios educativos a igualdad de otras condiciones. En este sentido, las mujeres salen más reforzadas que los chicos. Si bien la relación entre logro educativo y nivel educativo de los padres está ampliamente contrastada en la literatura internacional, este artículo permite ampliar la mirada de dicha relación hacia las trayectorias académicas.

Los niveles altos de apoyo parental contribuyen en mayor medida a facilitar la continuidad en los estudios de los jóvenes que el apoyo que proporcionan los profesores o tutores, pero no encontramos evidencias significativas de estos efectos entre chicos y chicas. Hay que puntualizar que una forma de desentranar la importancia del apoyo parental hubiera sido segregando el que realiza la madre del que realiza el padre, orientado a resolver el efecto de transmisión intergeneracional de género, pero los datos no permiten dicha diferenciación. La mayoría de estudios que analizan la influencia del apoyo parental no realizan dicha segmentación (básicamente porque los datos no suelen recogerla), como también sucede en el caso de los profesores. Sería interesante poder analizar la influencia acreditada del apoyo parental desde una perspectiva de transmisión intergeneracional de género. Otra posible limitación de la variable de apoyo parental empleada, como también sucede en el apoyo de una figura docente, es que se trata de una variable que mide la percepción del entrevistado sobre una realidad en el pasado preguntada de forma un tanto amplia. Algunos estudios utilizan una batería de ítems en las encuestas para determinar de forma más detallada el nivel de apoyo parental (como es el caso de la edición de PISA 2009).

Los análisis utilizados en esta investigación permiten identificar tres grupos de trayectorias más o menos homogéneas internamente, pero no permiten examinar las trayectorias menos convencionales por sí solas ni los factores que se les asocian. En este sentido, sería interesante realizar análisis de tipo cualitativo para examinar los factores que están relacionados con trayectorias menos convencionales, como por ejemplo el reingreso a la formación (Nobile, 2016), y en qué medida hay algún tipo de diferencia según género.

Otro elemento importante a tener en cuenta es que las características contextuales del mercado de trabajo pueden influir en los itinerarios académicos de los jóvenes. El periodo de trayectorias analizado en esta investigación coincide en gran parte con una etapa de expansión económica en España, caracterizada por una mayor demanda de mano de obra poco cualificada en el sector de la construcción. En ese periodo, permanecer en el sistema educativo suponía, para algunos jóvenes, un coste de oportunidad alto, ya que el mercado de trabajo ofrecía mayores beneficios. Es de suponer que las variaciones laborales hayan influenciado en cierta medida en la toma de decisiones de los jóvenes y en sus 
itinerarios académicos. Asimismo, en futuros análisis, sería apropiado plantear qué implicaciones tienen las trayectorias educativas en los itinerarios del mercado de trabajo, para poder observar en qué medida las primeras condicionan a los segundos y las variaciones respecto al género.

Los resultados de esta investigación señalan que las trayectorias educativas de los jóvenes dependen en gran medida de factores exógenos a las escuelas, como son el capital social de las familias o las características sociodemográficas de los individuos. A pesar de ello, tanto en Cataluña como en España, las políticas educativas se han concentrado principalmente en atender a factores intraescolares que ejercen una influencia limitada en la reducción de las desigualdades académicas.

\section{Referencias bibliográficas}

Aassve, A.; Billari, F.C. y PiccarReta, R. (2007). «Strings of adulthood: A sequence analysis of young British women's work-family trajectories». European Journal of Population / Revue européenne de Démographie, 23 (3-4), 369-388. $<$ http://dx.doi.org/10.1007/s10680-007-9134-6>

Aввотt, A. y Tsay, A. (2000). «Sequence Analysis and Optimal Matching Methods in Sociology». Sociological Methods \& Research, 29, 3-33. $<$ http://dx.doi.org/10.1177/0049124100029001001>

Billari, F.C. (2001). «Sequence Analysis in Demographic Research and Applications». Canadian Studies in Population, 28, 439-58.

BozicK, R. y DeLuCA, S. (2005). «Better late than never?: Delayed enrollment in the high school to college transition». Social Forces, 84 (1), 527-550. $<$ http://dx.doi.org/10.1353/sof.2005.0089>

Buchmann, C.; Diprete, Th.A. y McDaniel, A. (2008). "Gender Inequalities in Education». Annual Review of Sociology, 34, 319-337. $<$ http://dx.doi.org/10.1146/annurev.soc.34.040507.134719>

Calero, J. y ChoI, A. (2009). «Determinantes del rendimiento educativo del alumnado de origen nacional e inmigrante en PISA-2006». Cuadernos Económicos del ICE, 78, 281-310.

CALERO, J. y WAisGRAIS, S. (2008). «QQué determina el rendimiento de los alumnos inmigrantes?: Una primera aproximación a partir de PISA-06». Investigaciones de Economía de la Educación, 3, 499-508.

CoOley, S. (1995). Suspension/Expulsion of Regular and Special Education Students in Kansas: A Report to the Kansas State Board of Education. Topeka: Kansas State Board of Education.

DaVIS-KeAN, P.E. (2005). "The influence of parent education and family income on child achievement: The indirect role of parental expectations and the home environment». Journal of Family Psychology, 19 (2), 294. $<$ http://dx.doi.org/10.1037/0893-3200.19.2.294>

DesJaRDins, S.L.; AhLBURG, D.A. y MCCAlL, B.P. (2006). «The effects of interrupted enrollment on graduation from college: Racial, income, and ability differences». Economics of Education Review, 25 (6), 575-590. $<$ http://dx.doi.org/10.1016/j.econedurev.2005.06.002>

DiPrete, T.A. y BuChmann, C. (2013). The rise of women: The growing gender gap in education and what it means for American schools. Nueva York: Russell Sage Foundation. 
DucKworth, A.L. y Seligman, Martin E.P. (2006). "Self-discipline gives girls the edge: Gender in self-discipline, grades, and achievement test scores». Journal of Educational Psychology, 98 (1), 198-208. <http://dx.doi.org/10.1037/0022-0663.98.1.198>

Entwisle, D.R.; Alexander, K.L. y Olson, L.S. (2007). «Early Schooling: The Handicap of Being Poor and Male». Sociology of Education, 80, 114-138. <http://dx.doi.org/10.1177/003804070708000202>

Escandell, X.; Mari-Klose, P. y Mari-Klose, M. (2015). «Gender Gaps in Educational Outcomes Among Children of New Migrants: The Role of Social Integration from a Comparative Perspective». Sociology Compass, 9 (12), 1036-1048. <DOI: $10.1111 /$ soc4.12328>

EUROSTAT. Glossary: Early leavers from education and training. <http://ec.europa.eu/ eurostat/statistics-explained/index.php/Glossary:Early_leaver_from_education_ and_training $>$.

Farkas, G.; Grobe, R.; Sheehan, D. y Shuan, Y. (1990). "Cultural Resources and School Success: Gender, Ethnicity, and Poverty Groups within an Urban School District». American Sociological Review, 55, 127-142. <http://dx.doi.org/10.2307/2095708>

Featherman, D.L. y CarTer, T.M. (1976). «Discontinuities in schooling and the socioeconomic life cycle». En: SEWell, W.H.; Hauser, R.M. y FEATHERMan, D.L. (eds.). Schooling and achievement in American society. Nueva York: Academic Press, 133-160.

Feliciano, C. y Rumbaut, R.G. (2005). «Gendered paths: Educational and occupational expectations and outcomes among adult children of immigrants». Ethnic and Racial Studies, 28 (6), 1087-1118. <http://dx.doi.org/10.1080/01419870500224406>

Fernández Enguita, M.; Mena Martínez, L. y Riviere Gómez, J. (2010). Fracaso y abandono escolar en España. Barcelona: Fundación "La Caixa".

Freeman, C.E. (2004). Trends in Educational Equity for Girls and Women. Washington, DC: Natl. Cent. Educ. Stat.

Gabadinho, A.; Ritschard, G.; Müller, N.S. y Studer, M. (2011). «Analyzing and Visualizing State Sequences in R with TraMineR». Journal of Statistical Software, $40(4), 1-37$.

Gabadinho, A.; Ritschard, G.; Studer, M. y Müller, N.S. (2009). «Mining Sequence Data in R with the TraMineR package: A User's Guide». Department of Econometrics and Laboratory of Demography. Ginebra: University of Geneva.

Gregory, J.F. (1996). "The crime of punishment: Racial and gender disparities in the use of corporal punishment in the U.S. Public Schools». Journal of Negro Education, 64, 454-462. <http://dx.doi.org/10.2307/2967267>

Hausmann, R.; Zahidi, S.; Tyson, L.; Hausmann, R.; Schwab, K. y Tyson, L.D.A. (2009). The global gender gap report 2009. Ginebra: World Economic Forum.

Heckman, J.J.; Stixrud, J. y UrzuA, S. (2006). "The Effects of Cognitive and Noncognitive Abilities on Labor Market Outcomes and Social Behavior». Journal of Labor Economics, 24 (3), 411-482. <http://dx.doi.org/10.1086/504455>

Instituto Nacional de Evaluación Educativa (2014). Sistema estatal de indicadores de la educación 2014. Madrid: Ministerio de Educación, Cultura y Deporte. 
JACOB, B.A. (2002). «Where the boys aren't: Non-cognitive skills, returns to school and the gender gap in higher education». Economics of Education Review, 21 (6), 589-598. <http://dx.doi.org/10.1016/S0272-7757(01)00051-6>

JEYNES, W.H. (2005). "A meta-analysis of the relation of parental involvement to urban elementary school student academic achievement». Urban Education, 40 (3), 237-269. <http://dx.doi.org/10.1177/0042085905274540>

Kim, Y.; Sherraden, M.; Huang, J. y Clancy, M. (2015). «Child Development Accounts and Parental Educational Expectations for Young Children: Early Evidence from a Statewide Social Experiment». Social Service Review, 89 (1), 99-137. <http://dx.doi.org/10.1086/680014>

MARTíneZ GARCÍA, J.S. (2007). "Clase social, género y desigualdad de oportunidades educativas». Revista de Educación, 342, 287-306.

- (2011). "Género y origen social: Diferencias grandes en fracaso escolar administrativo y bajas en rendimiento educativo». Revista de la Asociación de Sociología de la Educación, 4 (3), 270-285.

MAU, W. (1997). «Parental influences on the high school students' academic achievement: A comparison of asian immigrants, asian americans, and white americans». Psychology in the Schools, 34 (3), 267-277.

$<$ http://dx.doi.org/10.1002/(SICI) 1520-6807(199707)34:3<267::AIDPITS9>3.0.CO;2-L>

MCBRIDE, B.A. y LIN, H. (1996). "Parental involvement in prekindergarten at-risk programs: Multiple perspectives». Journal of Education for Students Placed at Risk, 1 (4), 349-372. <http://dx.doi.org/10.1207/s15327671espr0104_5>

McFadden, A.C. II; Marsh, G.E.; Price, B.J. y Hwang, Y. (1992). «A study of race and gender bias in the punishment of handicapped school children». The Urban Review, 24 (4), 239-251. <http://dx.doi.org/10.1007/BF01108358>

Milesi, C. (2010). «Do all roads lead to Rome?: Effect of educational trajectories on educational transitions». Research in Social Stratification and Mobility, 28 (1), 23-44. <http://dx.doi.org/10.1016/j.rssm.2009.12.002>

MoffitT, T.E.; CasPi, A.; RutTer, M. y Silva, P.A. (2001). «Sex Differences in Antisocial Behavior: Conduct Disorder, Delinquency, and Violence in the Dunedin Longitudinal Study». Cambridge, UK: Cambridge University Press. <http://dx.doi.org/10.1017/CBO9780511490057>

Mortenson, T.G. (1999). "Where Are the Boys?: The Growing Gender Gap in Higher Education». College Board Review, 188, 8-17.

Muller, C. (1998). "Gender differences in parental involvement and adolescents' mathematics achievement». Sociology of Education, 71 (4), 336-356. <http://dx.doi.org/10.2307/2673174>

Nobile, M. (2016). «Los egresados de las Escuelas de Reingreso: Sobre los soportes mínimos para aprovechar una política de reinserción educativa». Espacios en Blanco: Revista de Educación. Serie Indagaciones, 26 (2).

Portes, A. y Rumbaut, R.G. (2001). Legacies: The Story of the Immigrant Second Generation. University of California Press y Russell Sage Foundation.

Rosenbaum, J. (2001). Beyond College for All: Career Paths for the Forgotten Half. Nueva York: Russell Sage. 
RousseEuw, P.J. (1987). «Silhouettes: A graphical aid to the interpretation and validation of cluster analysis». Journal of Computational and Applied Mathematics, 20, 53-65. <http://dx.doi.org/10.1016/0377-0427(87)90125-7>

Rubie-DAvies, C. (2007). «Classroom interactions: Exploring the practices of highand low-expectation teachers». British Journal of Educational Psychology, 77 (2), 289-306.

<http://dx.doi.org/10.1348/000709906X101601>

- (2010). "Teacher expectations and perceptions of student attributes: Is there a relationship?». British Journal of Educational Psychology, 80 (1), 121-135. <http://dx.doi.org/10.1348/000709909X466334>

SANKOFF, D. y KRUSKAL, J.B. (eds.) (1983). Time warps, string edits and macromolecules. Reading, MA: Addison Wesley.

SEWELL, W.H. y SHAH, V.P. (1968). «Parents' education and children's educational aspirations and achievements». American Sociological Review, 191-209. <http://dx.doi.org/10.2307/2092387>

Skiba, R.J.; Michael, R.S.; NARdo, A.C. y Peterson, R.L. (2002). «The color of discipline: Sources of racial and gender disproportionality in school punishment». The Urban Review, 34 (4), 317-342. <http://dx.doi.org/10.1023/A:1021320817372>

SKibA, R.J.; PETERSON, R.L. y Williams, T. (1997). «Office referrals and suspension: Disciplinary intervention in middle schools». Education and Treatment of Children, 20 (3), 295-315.

Smith, J.R.; Brooks-GunN, J. y Klebanov, P.K. (1997). "Consequences of living in poverty for young children's cognitive and verbal ability and early school achievement». En: DunCAN, G.J. y BROOKS-GunN, J. (eds.). Consequences of growing up poor. Nueva York: Russell Sage Foundation, 132-189.

SoKAL, L.; KATZ, H.; ChaszewSKI, L. y WojICK, C. (2007). «Good-bye, Mr. Chips: Male teacher shortages and boys' reading achievement». Sex Roles, 56 (9-10), 651659. <http://dx.doi.org/10.1007/s11199-007-9206-4>

SORHAGEN, N.S. (2013). "Early teacher expectations disproportionately affect poor children's high school performance». Journal of Educational Psychology, 105 (2), 465-477. <http://dx.doi.org/10.1037/a0031754>

Troiano, H. y DAZA, L. (2016). "La transició cap a la universitat». En: Equitat en l'accés $i$ en la inserció professional dels graduats universitaris. Barcelona: AQU Catalunya.

WARD, J.H. Jr. (1963). «Hierarchical Grouping to Optimize an Objective Function». Journal of the American Statistical Association, 58, 236-244. <http://dx.doi.org/10.1080/01621459.1963.10500845>

Weinstein, R.S. y MCKOwn, C. (1998). "Expectancy effects in "context”: Listening to the voices of students and teachers». En: BROPHY, J. (eds.). Advances in research on teaching: Expectations in the classroom. Greenwich, CT: JAI Press, 215-242.

Zhu, S.; Tse, S.; Cheung, S.H. y Oyserman, D. (2014). "Will I get there?: Effects of parental support on children's possible selves». British Journal of Educational Psychology, 84 (3), 435-453. <http://dx.doi.org/10.1111/bjep.12044> 


\section{Apéndice}

Gráfico A.1. Tiempo medio en cada estado educativo por conglomerados de itinerarios educativos (2 clústeres)

Cluster 1

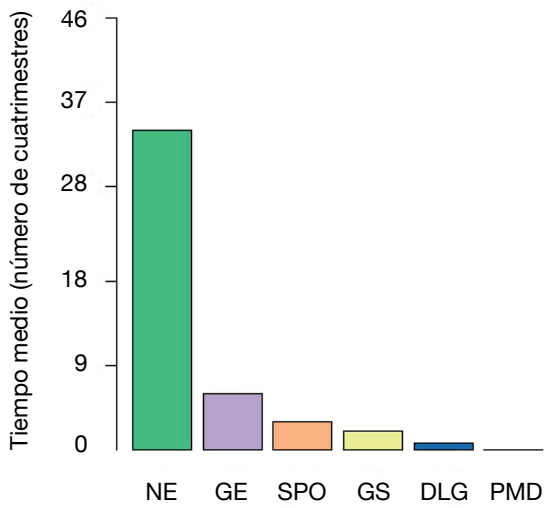

Cluster 2

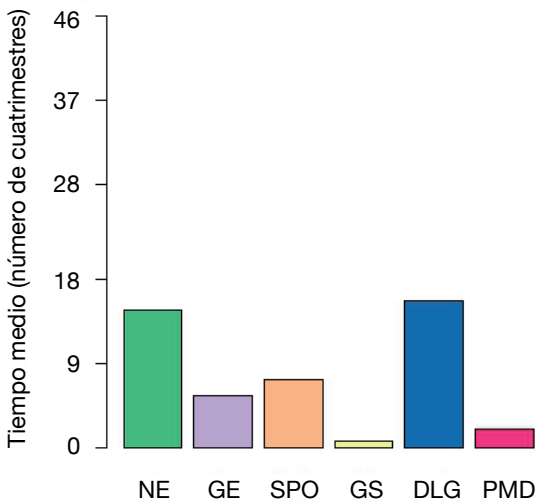

$$
\begin{aligned}
& \square \mathrm{NE} \square \mathrm{SPO} \square \mathrm{DLG} \\
& \square \mathrm{GE} \square \mathrm{GS} \square \mathrm{PMD}
\end{aligned}
$$

Fuente: elaboración propia a partir de EJC12.

Gráfico A.2. Tiempo medio en cada estado educativo por conglomerados de itinerarios educativos (2 clústeres)
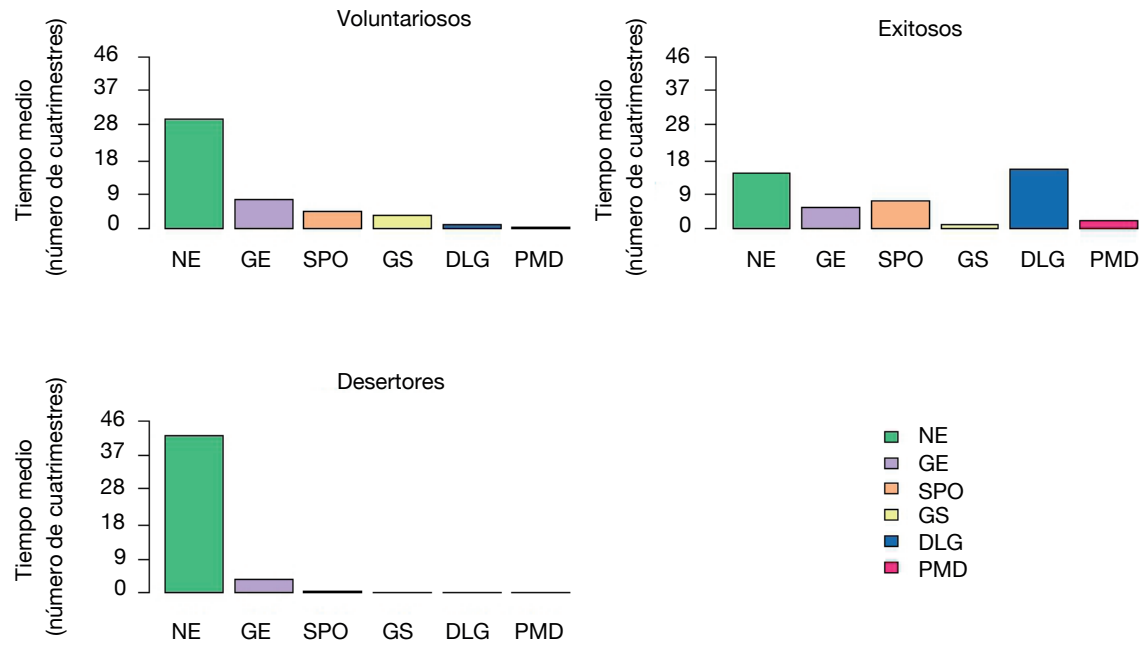

Fuente: elaboración propia a partir de EJC12. 